\title{
Exploring the Effects of Performance Feedback on Motivational States
}

\author{
Matthew Castillo, Hudson Taylor, \& Mitzi Desselles \\ Louisiana Tech University \\ Ann-Marie Castille \\ Nicholls State University
}

\begin{abstract}
This study explored whether performance feedback induces certain motivational states. If managers can induce states that are ideal in work situations by providing different types of feedback messages, then they could facilitate enhancing their employees' motivation. After completing a task and receiving a random feedback message, 799 participants recorded their motivational state. Interaction effects were hypothesized between feedback valence (positive vs negative) and accuracy (accurate vs inaccurate) with the conforming-rebellious and crossed transactional pairs. We expected that negative, inaccurate feedback would increase the frequencies of the rebellious and self-sympathy states while all other feedback conditions (negativeaccurate, positive-accurate, and positive-inaccurate) would increase the frequencies of the conforming and self-mastery states. We also expected that positive feedback would increase the frequency of the paratelic state, negative feedback would increase the frequency of the telic state, accurate feedback would increase the frequency of the conforming state, and inaccurate feedback would increase the frequency of the rebellious state. Multinomial log-linear analysis revealed no interaction effect between feedback valence and accuracy on motivational state but did support main effect hypotheses. Positive feedback increased frequencies of the paratelic, conforming, and other-mastery states. Negative feedback increased frequencies of the telic, rebellious, self-sympathy, and self-mastery states. Overall, the effects of performance feedback on motivational states are different depending on the type of feedback message, which may help explain why feedback does not always result in desired behavior change.
\end{abstract}

Keywords: Reversal Theory, motivation, performance, reactions to supervisor feedback

Performance appraisals are designed to provide constructive feedback to employees to increase their commitment to and performance in their organization (Aguinis, 2013). The purpose of performance feedback is to align individual and organizational goals as well as clarify expectations between employees, their leaders, and the organization as a whole (Aguinis, 2009). However, performance feedback has been criticized because it is often demotivating and can lead to adverse outcomes when not handled correctly, such as decreases in employee motivation and job satisfaction, as well as increases in turnover and burnout (Mueller-Hanson \& $\mathrm{Pu}-$ lakos, 2015; Ryu \& Hong, 2019). Longenecker and Goff (1992) suggest that formal appraisal systems frustrate managers and employees because of problems associated with

Editorial Board Invited Paper from the 2019 International Reversal Theory Conference, Las Vegas, New Mexico

Matthew Castillo, Hudson Taylor, \& Mitzi Desselles, Psychology \& Behavioral Sciences, Louisiana Tech University; Ann-Marie Castille, Business Administration \& Computer Information Systems, Nicholls State University

Correspondence concerning this article should be addressed to Matthew Castillo. E-mail: msc044@latech.edu their design and implementation. Specifically, previous research has focused on feedback as a critical communication activity (O'Reilly \& Anderson, 1980). Managers may not like giving bad news or having difficult conversations with employees. They also may not be effective communicators of performance information. From an employee's perspective, individuals may not perceive the feedback they receive as accurate or may have negative reactions to receiving negative feedback (Steelman \& Rutkowski, 2004). Performance appraisal effectiveness depends on needs, expectations, and perceptions of managers and direct reports including the purpose of appraisal, the level of fairness, and the completeness of communication when employees are given feedback on their performance (Longenecker \& Goff, 1992). Feedback results in immediate, and sometimes automatic, reactions in employees that can lead to various outcomes (Aguinis et al., 2012). For example, researchers have found that negative feedback tends to lead to negative emotional reactions as well as a decreased probability that an individual will improve performance in the future (Ilgen \& Davis, 2000; Ilies et al., 2007). However, other findings suggest that negative feedback can inspire individuals to learn from their mistakes and correct them (Podsakoff \& Farh, 1989). These conflicting results highlight the need to further explore how recipients perceive and respond to feedback messages to clarify 
the outcomes of certain types of feedback and determine the best way to deliver performance feedback to different people in different situations.

Motivational theories could provide an explanation for the impact of feedback on performance. Although previous research (Ilgen et al., 1979; Ilies \& Judge, 2005; Kinicki et al., 2004; Kluger et al., 1994) has explored reactions to performance feedback (i.e., affective, cognitive, behavioral), little attention has been paid to the motivational states of recipients following feedback. That is, an individual's immediate motivational experience in relation to the feedback message they receive and how they respond to the feedback in terms of their desired behavior. Employees' reactions to performance feedback could affect their future performance (Aguinis et al., 2012; Kluger \& DeNisi, 1996). As such, managers could benefit from understanding the relationships between performance feedback messages and motivational states to determine the types of feedback that should be given in abundance versus avoided when possible. For the present study, we explored associations between various performance feedback messages and motivational states. Specifically, our research question was: Does performance feedback induce certain motivational states? The purpose of our exploratory study was to discover if there is a relationship between different types of performance feedback messages and an individual's resulting motivational state according to reversal theory (Apter, 2007). This is important because previous research supports that certain states may result in higher performance for certain jobs. For example, Barr et al. (1993) found that individuals in the paratelic state may solve complex tasks more easily. Apter (2001) asserts that the conforming state is ideal for work requiring compliance with regulations and routines. Finally, researchers purport that the rebellious and paratelic states may be necessary for performing innovative tasks (Dixon, 1993a, 1993b; Griffin \& McDermott, 1998). As such, managers in organizations who are responsible for providing performance feedback to employees would benefit from knowledge of various characteristics of feedback messages resulting in an increased frequency of certain states. Further, managers may be able to encourage maintenance in a state or reversals to opposing states by providing certain kinds of feedback.

The present study aims to explore what types of feedback increase frequencies in which reversal theory states. Specifically, our study focused on performance feedback valence and accuracy to determine if there are differences in motivational states after receiving positive versus negative and accurate versus inaccurate feedback. ${ }^{1}$ Accurate feedback refers to a feedback message that reflects an individual's actual performance on the task while inaccurate feedback refers to a feedback message that does not reflect an individual's actual performance on the task. Based on previous knowledge of an individual's motivation ${ }^{2}$ while in different reversal theory states, and potential antecedents of that type of motivation based on valence and accuracy of performance feedback, the following hypotheses were formed:

H1: a) Positive feedback will increase the frequency of the paratelic state, compared to negative feedback. b) Negative feedback will increase the frequency of the telic state, compared to positive feedback.

H2: a) Accurate feedback will increase the frequency of the conforming state, compared to inaccurate feedback. b) Inaccurate feedback will increase the frequency of the rebellious state, compared to accurate feedback.

H3: There will be an interaction between valence and accuracy of feedback, such that a) negative-inaccurate feedback will increase the frequency of the rebellious state, compared to negative-accurate, positive-accurate, or positive-inaccurate feedback; b) negative-accurate, positiveaccurate, and positive-inaccurate feedback will increase the frequency of the conforming state; c) negative-inaccurate feedback will increase the frequency of the self-sympathy state, compared to negative-accurate, positive-accurate, or positive-inaccurate feedback; and d) negative-accurate, positive-accurate, and positive-inaccurate feedback will increase the frequency of the self-mastery state.

Employees who receive positive feedback (i.e., praise such as "Good job. You have reached your goal.") are expected to be more likely to be in a playful, paratelic state as opposed to a serious, telic state (hypothesis 1a). The theoretical argument is that recipients of multiple positive feedback messages may experience a contingent reversal from the telic to the paratelic state (Apter, 2001, 2005). This is likely due to recipients' perception of the feedback as valid, regardless of whether it actually is valid (cf. fundamental attribution error, Gawronski, 2007) and therefore it should be accepted and internalized. In practical terms, positive feedback that triggers the paratelic state may result in greater enjoyment of the task itself and lead to higher performance. This positive emotion may create a halo effect and lead to feelings of enjoyment of the work itself. Enjoyment of the task may result in better performance and increase the likelihood the recipient goes above and beyond (i.e., increase discretionary organizational citizenship behaviors (OCBs); Organ, 1988). In contrast, employees who receive negative feedback (i.e., criticism such as "Unfortunately, you failed to reach your goal.") should be more likely to be in a serious (telic) state of mind (hypothesis 1b). The theoretical argument is that recipients of multiple negative feedback messages may persist in the

\footnotetext{
${ }^{1}$ The authors note that the use of the phrases positive and negative feedback is different in this work than in the operant conditioning literature.

${ }^{2}$ The focus of this article is on motivational states, not the alternative ways of experiencing each state (i.e., each state within a pair may be experienced positively (satisfied) or negatively (not satisfied)).
} 
telic state because reaching the goal is important, and they continue working in order to reach the goal and experience the satisfaction of doing so (Apter, 2005; Cron et al., 2005). This hypothesis is consistent with control theory, which suggests that reactions to performance feedback depend on a desire to minimize a goal-performance discrepancy (Carver \& Scheier, 1981). In the telic state, employees would focus on attaining their performance goal, which they learned through the negative feedback message they had not achieved (Apter, 2007; Krenn et al., 2013).

With respect to the hypotheses on feedback accuracy, employees who receive accurate feedback are expected to be more likely to adhere to organizational rules (hypothesis 2a) while employees who receive inaccurate feedback are more likely to be motivated by personal freedom and thus be in the rebellious state (hypothesis $2 \mathrm{~b}$ ). Most would agree that accurate feedback is what is expected from supervisors (Aguinis, 2013). Accurate feedback may be accompanied by an implicit agreement between giver and recipient that when the feedback is consistent with reality, the recipient is expected to accept it and adjust their performance accordingly (Aguinis, 2009). As a result, accurate feedback is expected to provoke the conforming state of mind. Inaccurate feedback violates this implicit agreement (i.e., breaks "the rules") as it provides information that is inconsistent with what actually happened. Therefore, inaccurate feedback is expected to induce a rebellious, rule-breaking state of mind in recipients (Apter, 2001; Jawahar, 2010; Steelman \& Rutkowski, 2004). The remaining hypotheses predict an interaction between valence and accuracy on the conforming-rebellious pair and the crossed transactional pairs (i.e., the four mutually exclusive combinations of the self and other pair crossed with the mastery and sympathy pair) (Apter, 2007).

Inaccurate feedback that is also negative is likely to be perceived as unjust or unfair criticism and is expected to result in greater frequencies of the rebellious state (hypothesis 3a). Negative-inaccurate feedback resulting in the rebellious state is concerning because it could give rise to counterproductive work behaviors (CWBs), such as sabotage, absenteeism, or other forms of employee withdrawal (Spector et al., 2006). Recipients of negative-inaccurate feedback may also feel wronged by the mischaracterization of their performance, such that they experience the self-mastery state as "losing" and through the mechanism of frustration, reverse to the self-sympathy state (in addition to rebellious - hypothesis 3c; Apter, 2001, 2005).

In contrast, employees who receive feedback that is negative and accurate, positive and accurate, or positive and inaccurate are expected to be in the conforming and self-mastery states. All three groups are expected to be motivated by doing what is expected (hypothesis $3 \mathrm{~b}$ ) and motivated by competence and control (hypothesis 3d). Employees who receive feedback that is negative and accurate are likely to feel that they need to work harder to perform well so as to successfully meet performance expectations; therefore, these employees would likely be in the conforming, self-mastery state though unsatisfied in that state. Employees who receive feedback that is positive and accurate are more likely to perceive their feedback as a legitimate confirmation that they did what was expected and are competent and capable at performing the task; therefore, these employees would likely be in the conforming, self-mastery state and feel satisfied in that state. Finally, employees who receive feedback that is positive and inaccurate are likely to accept the feedback at face value and not dispute it (i.e., conforming state) as well as conclude they succeeded (i.e., experience the self-mastery state as gaining). Feedback recipients' tendency to accept positive feedback regardless of its accuracy has been referred to as the "credibility gap" and supported in the literature (Halperin et al., 1976; Jacobs et al., 1973; Johnson \& Nawrocki, 1967.)

\section{Method}

\section{Participants}

The sample included 800 individuals who were 18 years of age and above, native English speakers, and residents of the U.S. or Canada. Participants were recruited from Amazon's Mechanical Turk (MTurk), a crowdsourcing marketplace and participant recruitment system. They were paid $\$ 1.60$ for their participation, which was based on a rate of $\$ 6.40$ per hour with the survey lasting approximately $15 \mathrm{~min}$ utes. One case was removed due to incomplete data resulting in a final sample of 799 .

\section{Measures}

Reversal theory states were measured using the Bundled Reversal Theory State Measure (RTSM - Bundled, Desselles et al., 2014). The bundled RTSM assesses frequencies of states in all possible reversal theory domains. Each reversal theory state (telic, paratelic, conforming, rebellious, selfmastery, other-mastery, self-sympathy, and other-sympathy) was treated as its own variable and data was coded as being present or absent in each state based on scores for each domain.

\section{Procedure}

After reading an overview of the study on the MTurk marketplace, participants clicked a link to the survey that was administered via the web-based survey tool Qualtrics. Individuals were deceptively told that the researchers needed help verifying the numbers of RGB codes for building a university information website for incoming college students. RGB refers to a system for representing color values on a computer display, specified by the combination of various proportions of red, green, and blue. Each parameter defines the intensity of the color as an integer between 0 and 225 . 
Then, participants completed a trial task and proceeded to set a goal for a three-minute task involving gathering RGB codes of primary colors from various specified Universities. The goal was in regard to either the number of universities for which they would provide RGB codes or the percentage of participants they would outperform. After completing the task, participants were shown a random positive or negative feedback message that was either accurate or inaccurate. Their motivational states were measured immediately following feedback.

The feedback message was considered positive or negative based on whether the individual was randomly told they reached or failed to reach their goal (feedback valence). The positive feedback message stated "Good job! You have reached your goal. You have identified the primary color RGB codes for $\{\#\}$ universities." or "Good job! You have reached your goal. You performed better than $\{\# \%\}$ of the participants." The negative feedback message stated "Unfortunately, you failed to reach your goal. You failed to identify the primary color RGB codes for $\{\#\}$ universities." or "Unfortunately, you failed to reach your goal. You failed to perform better than $\{\# \%\}$ of the participants." Randomization occurred via survey flow logic added to the survey instrument in the survey platform Qualtrics.

Feedback accuracy could not be randomly assigned because it is dependent upon participants' actual performance on the task compared to their feedback valence condition, therefore, the feedback message was coded as accurate or inaccurate based on whether the feedback valence matched the participants' performance (whether or not they reached their goal). For instance, if a participant reached their goal but received negative feedback stating that they failed to reach their goal, their feedback was inaccurate.

\section{Data Analysis}

The data were analyzed using log-linear analysis following the steps outlined by Agresti (2007), which will be described in more detail below. Log-linear analysis was conducted via the statistical software R using the "gmodels" and "MASS" packages for fitting log-linear models (Venables \& Ripley, 2002; Warnes et al., 2018) to determine the frequencies and percentage of individuals in each motivational state as a function of the type of feedback message they received. Log-linear analysis is similar to the Chi-square test of independence in that it compares expected to observed frequencies among categorical variables to determine which model of expected frequencies best fits the obtained data. Unlike the chi-square test, log-linear analysis allows comparison among three or more categorical variables at a time (Tabachnick \& Fidell, 2013).

A key issue in conducting log-linear analysis is how to choose the model that most optimally fits the data (Howell, 2010). If there is enough evidence to reject the null hypothe- sis, then one can conclude the model does not fit the data and the interaction term is necessary since the variables are not independent (i.e., mutual dependence; Howell, 2010). For more complex designs in log-linear analysis, there are several potential alternatives to the saturated, or mutual dependence, model that includes all possible parameters and fits the data perfectly. The researcher must compare alternative models to determine if interaction effects are significant, since they are not directly tested in the analysis, and decide which model is the most parsimonious. The purpose of fitting the perfect saturated model to the obtained data is to reveal nonsignificant effects that might identify simpler models with similar fit (Howell, 2010).

To find a model with good fit and a simple structure out of all possible models, we started by looking at the log-linear analysis output of all likelihood ratio and Pearson chi-square values with their respective $p$ values (Howell, 2010). We began with the saturated model containing all possible effects and used a backward elimination process to remove terms starting with the highest-order interactions (usually four- or three-way interactions), then lower-order (two-way) interactions (Agresti, 2007). The saturated model includes associations between explanatory variables assuming conditional independence for all other paired associations. To build the saturated model, we added all homogeneous associations (twofactor terms) followed by all three-factor terms. If there is no improvement in fit after adding three-factor terms, then models without three-factor terms should be considered. Since this was the case, we started removing two-factor terms that did not make significant contributions to the homogeneous association model using a backward elimination process to take out terms with the smallest change in likelihood ratio chi-square $\left(\Delta G^{2}\right.$; Agresti, 2007). We then identified all models that were non significant with $p>.05$. These are the models that should be considered because their estimated cell frequencies do not differ significantly from the obtained frequencies. Significant models with $p<.05$ do not adequately fit the data due to substantial differences between observed and expected frequencies. We compared the likelihood ratio chi-square value of each non-significant model with all of the models above it (containing lower-order interactions and main effects) until a comparison resulted in a statistically significant new model indicating that dropping any other component from that model leads to worse fit (Howell, 2010).

\section{Results and Discussion}

First, we fit the saturated model assuming conditional independence. Then we added all homogeneous associations (two-factor terms). Next we added all three-factor terms. At this stage, we determined that there was no improvement in fit, so models without three-factor terms were considered. After that, we removed two-factor terms that did not make a significant contribution to the homogeneous associa- 
Table 1

\begin{tabular}{lrrrr} 
Hierarchical Log-Linear Analysis Summary & Output of Model Statistics \\
\hline Model & $G^{2}$ & $d f$ & $\Delta G^{2}$ & $d f$ \\
\hline Mutual Independence + Val*Acc & 181.98 & 55 & - & - \\
Homogeneous associations & 50.37 & 38 & - & - \\
PT*Val & 66.77 & 39 & 16.40 & 1 \\
PT*Acc & 51.19 & 39 & 0.82 & 1 \\
CR*Val & 67.31 & 39 & 16.94 & 1 \\
CR*Acc & 59.94 & 39 & 4.57 & 1 \\
Cross*Val & 73.93 & 41 & 23.56 & 3 \\
Cross*Acc & 51.98 & 41 & 1.61 & 3 \\
Drop PT*Acc \& Cross*Acc & 53.01 & 42 & 1.82 & 3 \\
Drop PT*Acc, Cross*Acc, \& CR*Acc & 57.09 & 43 & 4.08 & 1 \\
\hline
\end{tabular}

Note. $\mathrm{PT}=$ paratelic/telic $; \mathrm{CR}=$ conforming/rebellious; Cross = crossed pairs; $\mathrm{Val}=$ valence $;$ Acc $=$ accuracy. The shaded row indicates the model retained.

tion model, using a backward elimination process to take out terms with the smallest change in likelihood ratio chi-square. This resulted in a retained model with the following interactions of interest (including main effects): paratelic/telic $\mathrm{x}$ valence, conforming/rebellious $\mathrm{x}$ valence, crossed pairs $\mathrm{x}$ valence, and conforming/rebellious $\mathrm{x}$ accuracy (see Table 1 for a summary of the results).

Note that there was no interest in interactions among reversal theory state pairs, only among states and feedback types. Interactions between paratelic/telic $\mathrm{x}$ accuracy and crossed pairs $x$ accuracy were the two-factor terms that were removed since they had the smallest change in likelihood ratio chi-square and did not contribute significantly to the model. After retaining the model, observed frequencies were plotted in a bar graph to visualize significant associations.

Results supported hypotheses $1 \mathrm{a}$ and $1 \mathrm{~b}$ in that positive feedback increased the frequency of the paratelic state and negative feedback increased the frequency of the telic state (see Figure 1). Learning that a goal was reached after reading a positive feedback message motivated the participants to focus on enjoying the task at hand rather than achievement. The support for hypothesis 1a suggests that managers should find opportunities to provide positive feedback whenever possible, since positive feedback may induce the paratelic state, which has been shown to improve performance and creativity (Dixon, 1993a). Indeed, this finding is in line with recommendations in the performance feedback literature regarding effective approaches to feedback delivery for improving employee well-being and engagement (Aguinis et al., 2012). For example, Bouskila-Yam \& Kluger (2011) suggest providing at least three pieces of positive feedback for every piece of negative feedback.

Given that the focus of the telic state is on goal attainment (Apter, 2001), the support for hypothesis 1b indicates that recipients of negative feedback suggesting that they did not reach their goal will subsequently be more motivated by goal attainment rather than enjoyment of the task. Based on this finding, if managers' intentions are to motivate their employees to reach an unmet goal, negative feedback may be beneficial for meeting those intentions. When providing negative feedback, managers should illustrate for employees how they can progress toward goal achievement (Hattie \& Timperley, 2007; Krenn et al., 2013). That is, managers should closely link negative feedback to their employees' knowledge and skills in relation to the steps employees can take to improve their performance (Aguinis et al., 2012). This is important because attribution theory asserts that positive and negative performance feedback influence the evaluation of task-related stimuli (Korn et al., 2016). Employees tend to attribute positive feedback internally (e.g., to their own abilities) and negative feedback externally (e.g., to environmental factors).

Although not hypothesized, results further revealed that positive feedback increased the frequencies of the conforming and other-mastery states (see Figures 2 and 3). Such a finding suggests that positive feedback reinforces for employees the notion that they are performing in a manner that is expected of them. Given this finding, the authors further suggest that for jobs that require strict conformity to rules, managers should consider providing employees with positive feedback regarding rule compliance. Regarding the other-mastery finding, given the nature of the task (i.e., helping build a university informational website for students), it is possible that positive feedback made participants feel that they were successfully helping others with their efforts (Apter, 2005). Also not hypothesized, results revealed that negative feedback increased the frequencies of the rebellious, self-mastery, and self-sympathy states (see Figures 2 and 3). This finding suggests that in a task where participants' actions are intended to help others (as in this study's case), receiving negative feedback made participants more likely to focus on themselves rather than helping others, therefore entering the self-focused states (i.e., self-mastery or selfsympathy). Based on these findings, the authors suggest 


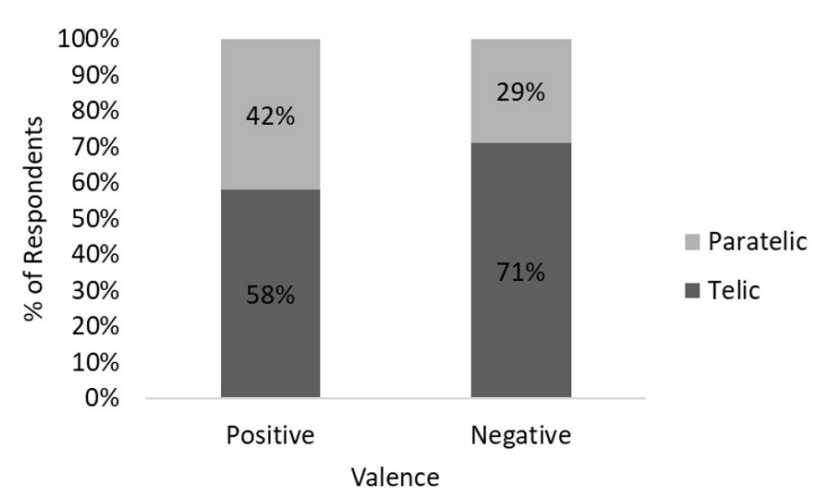

Figure 1. Percent of respondents in telic and paratelic states by feedback valence.

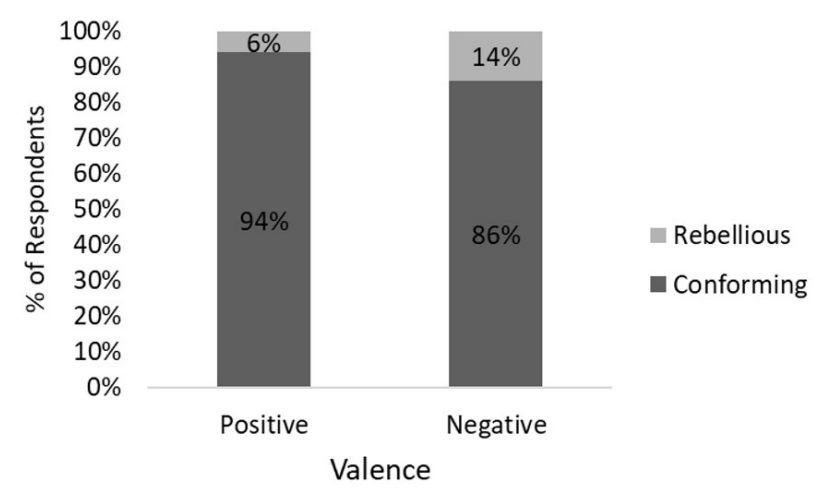

Figure 2. Percent of respondents in conforming and rebellious states by feedback valence.

that managers of employees in roles requiring helping others should consider providing sparse amounts of negative feedback.

Regarding the effects of feedback accuracy, hypotheses $2 \mathrm{a}$ and $2 b$ were not supported in that accurate feedback did not

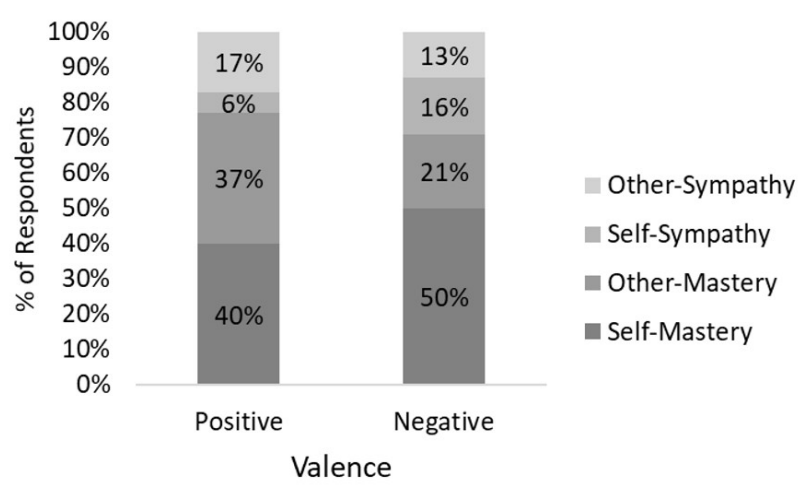

Figure 3. Percent of respondents in transactional pair states by feedback valence.

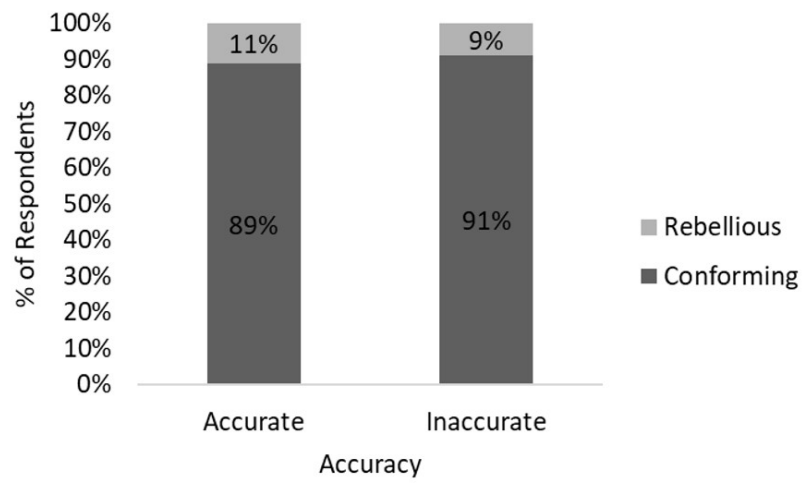

Figure 4. Percent of respondents in conforming and rebellious states by feedback accuracy.

increase the frequency of the conforming state compared to inaccurate feedback and inaccurate feedback did not increase the frequency of the rebellious state compared to accurate feedback (see Figure 4). Contrary to our hypotheses, inaccurate feedback increased the frequency of the conforming state relative to accurate feedback. The authors suspect such a finding may be due to the experimental nature of the task compared to the real world context of receiving inaccurate feedback on the actual performance of one's job. Given these results, the authors further suspect that participants likely experienced frustration in the conforming state rather than feelings of defiance in the rebellious state upon receiving inaccurate feedback. Although inconsistent with our hypotheses, the authors argue that these findings, in conjunction with the preponderance of evidence in the performance feedback literature, still suggest that managers should attend to the accuracy of the feedback that they are providing to employees and not just whether the information is positive or negative in its connotation. For example, Steelman \& Rutkowski (2004) found that supervisor credibility and feedback quality (i.e., perceived accuracy) are significant moderators of the relationship between unfavorable feedback and employee motivation to use the feedback for performance improvement.

There was no interaction effect of accuracy and valence on state, thus hypotheses 3a, 3b, 3c, and 3d were not supported. In summary, hypotheses $1 \mathrm{a}$ and $1 \mathrm{~b}$, regarding main effects of feedback valence and accuracy on motivational states were fully supported while hypotheses $2 \mathrm{a}-3 \mathrm{~d}$ regarding the main effect of feedback accuracy and interactions between feedback valence and accuracy were not supported. Observed frequencies were used to calculate the percent of respondents in cells to create graphical representations of the results, as shown in the figures.

Overall, the present study revealed that the effects of feedback on motivational state are different depending on whether the feedback is positive or negative and accurate or inaccurate, which may help explain why feedback does not 
always result in desired behavior change. Further, the experimental manipulation of feedback resulted in different states. Managers should take this into consideration when crafting feedback messages to provide to employees during formal performance reviews.

\section{Limitations and Future Directions}

This study was not without limitations. The feedback messages provided to participants were administered by a computer. As such, further research is needed to determine if the effects are generalizable to recipients of feedback from a person, particularly employees receiving performance feedback from their immediate supervisor. Also, participants' motivational state pre-feedback was not measured, therefore it is unclear if the manipulation of feedback triggered an actual reversal of states. Measuring motivational states preand post-feedback will allow the researchers to determine if feedback messages cause a reversal. Our study did not investigate the extent to which each motivational state was satisfied. Including an assessment of the satisfactions of the states in future research would address questions of whether participants experienced the states as gaining or losing after receiving the feedback. We also encourage future research examining affective states alongside motivational states to examine if the accuracy and valence of feedback messages trigger specific state-linked emotions.

\section{Conclusion}

Our study examined the frequency of psychological states following performance feedback that signaled failure versus attainment, accurately or inaccurately, on a short-term task goal. Results suggest that positive feedback induces the paratelic state and negative feedback induces the telic state. Managers could use information about reactions to feedback to encourage the states that are most beneficial for their direct reports to be in at work for their particular work situation. For instance, managers hoping to encourage creativity should consider providing an abundance of positive feedback. Managers of employees in roles requiring strict adherence to rules should consider taking extra precaution to ensure that their feedback messages are accurate.

\section{References}

Agresti, A. (2007). Loglinear models for contingency tables. In A. Agresti (Eds.), An introduction to categorical data analysis (pp. 204-243). Hoboken, NJ: Wiley.

Aguinis, H. (2009). Performance management (2nd ed.). Upper Saddle River, NJ: Pearson Prentice Hall.

Aguinis, H., Gottfredson, R. K., \& Joo, H. (2012). Delivering effective performance feedback: The strengths-based approach. Business Horizons, 55(2), 105-111.
Aguinis, H. (2013). Performance management (3rd ed.). Upper Saddle River, NJ: Pearson Prentice Hall.

Apter, M. J. (2001). Motivational styles in everyday life: A guide to reversal theory. Washington, DC: American Psychological Association.

Apter, M. J. (2005). Personality dynamics: Key concepts in reversal theory. Loughborough, UK: Apter International.

Apter, M. J. (2007). Reversal theory: The dynamics of motivation, emotion, and personality. Oxford: Oneworld Publications.

Barr, S. A., McDermott, M. R., \& Evans, P. (1993). Predicting persistence: A study of telic and paratelic frustration. In J. H. Kerr, S. Murgatroyd, \& M. J. Apter (Eds.), Advances in reversal theory (pp. 123-136). Amsterdam: Swets \& Zeitlinger.

Bouskila-Yam, O., \& Kluger, A. N. (2011). Strength-based performance appraisal and goal setting. Human Resource Management Review, 21(2), 137-147.

Carver, C. S., \& Scheier, M. F. (1981). The self-attentioninduced feedback loop and social facilitation. Journal of Experimental Social Psychology, 17, 545-568. doi: 10.1016/0022-1031(81)90039-1

Cron, W. L., Slocum, Jr, J. W., VandeWalle, D., \& Fu, Q. (2005). The role of goal orientation on negative emotions and goal setting when initial performance falls short of one's performance goal. Human Performance, 18(1), 5580.

Desselles, M. L., Murphy, S. L., \& Theys, E. R. (2014). The development of the reversal theory state measure. Journal of Motivation, Emotion, and Personality, 2, 10-21. doi: 10.12689/jmep.2014.202

Dixon, M. (1993a, October 27). Not brain-power, but frame of mind. Financial Times, p. 29.

Dixon, M. (1993b, October 6). The source of effective motivation. Financial Times, p. 14.

Field, A., Miles, J., \& Field, Z. (2012). Discovering statistics using $R$. Thousand Oaks, CA: Sage publications.

Gawronski, B. (2007). Fundamental attribution error. Encyclopedia of Social Psychology, 367-369.

Griffin, M., \& McDermott, M. R. (1998). Exploring a tripartite relationship between rebelliousness, openness to experience and creativity. Social Behavior and Personality: An International Journal, 26, 347-356. doi: 10.2224/sbp.1998.26.4.347

Halperin, K., Snyder, C. R., Shenkel, R. J., \& Houston, B. K. (1976). Effect of source status and message favorability on acceptance of personality feedback. Journal of Applied Psychology, 61(1), 85-88.

Hattie, J., \& Timperley, H. (2007). The power of feedback. Review of Educational Research, 77, 81-112.

Howell, D. C. (2010). Log linear analysis. In D. C. Howell (Ed.), Statistical methods for psychology (pp. 629-658). Belmont, CA: Cengage Wadsworth. 
Ikramullah, M., Shah, B., Hassan, F., Zaman, T., \& Khan, H. (2011). Fairness perceptions of performance appraisal system: An empirical study of civil servants in district Dera Ismail Khan. International Journal of Business and Social Science, 2, 92-100. Retrieved from https://ijbssnet.com/journals/Vol_2_No_21_Special_Issue _November_2011/12.pdf

Ilgen, D., \& Davis, C. (2000). Bearing bad news: Reactions to negative performance feedback. Applied Psychology, 49, 550-565. doi: 10.1111/1464-0597.00031

Ilgen, D. R., Fisher, C. D., \& Taylor, M. S. (1979). Consequences of individual feedback on behavior in organizations. Journal of Applied Psychology, 64, 349-371. doi: 10.1037/0021-9010.64.4.349

Ilies, R., DePater, I. E., \& Judge, R. (2007). Differential affective reactions to negative and positive feedback, and the role of self-esteem. Journal of Managerial Psychology, 22, 590-609. doi: 10.1108/02683940710778459

Ilies, R., \& Judge, T. A. (2005). Goal regulation across time: The effects of feedback and affect. Journal of Applied Psychology, 90, 453-467. doi: 10.1037/0021-9010.90.3.453

Jacobs, M., Jacobs, A., Feldman, G., \& Cavior, N. (1973). Feedback II-The "credibility gap": Delivery of positive and negative and emotional and behavioral feedback in groups. Journal of Consulting and Clinical Psychology, 41, 215-223. doi: 10.1037/h0035100

Jawahar, I. M. (2010). The mediating role of appraisal feedback reactions on the relationship between rater feedbackrelated behaviors and ratee performance. Group $\mathcal{F}$ Organization Management, 35(4), 494-526.

Johnson, W. A., \& Nawrocki, L. H. (1967). Effects of simulated social feedback on individual tracking performance. Journal of Applied Psychology, 51(2), 146-151.

Kinicki, A. J., Prussia, G. E., Wu, B., \& McKee-Ryan, F. M. (2004). A covariance structure analysis of employees' response to performance feedback. Journal of Applied Psychology, 89, 1057-1069. doi: 10.1037/00219010.89.6.1057

Kluger, A. N., Lewinsohn, S., \& Aiello, J. (1994). The influence of feedback on mood: Linear effects on pleasantness and curvilinear effects on arousal. Organizational Behavior and Human Decision Processes, 60, 279-299. doi: 10.1006/obhd.1994.1084

Kluger, A. N., \& DeNisi, A. (1996). The effects of feedback interventions on performance: A historical review, a metaanalysis, and a preliminary feedback intervention theory. Psychological Bulletin, 119(2), 254-284.

Korn, C. W., Rosenblau, G., Rodriguez Buritica, J. M., \& Heekeren, H. R. (2016). Performance feedback processing is positively biased as predicted by attribution theory. PloS one, 11(2), e0148581. doi: 10.1371/journal.pone.0148581
Krenn, B., Würth, S., \& Hergovich, A. (2013). The impact of feedback on goal setting and task performance: Testing the feedback intervention theory. Swiss Journal of Psychology, 72(2), 79-89.

Longenecker, C. O., \& Goff, S. J. (1992). Performance appraisal effectiveness: A matter of perspective. SAM Advanced Management Journal, 57, 17-23.

McHugh, M. L. (2013). The chi-square test of independence. Biochemia Medica, 23, 143-149. doi: 10.11613/BM.2013.018

Mueller-Hanson, R. A., \& Pulakos, E. D. (2015). Putting the "performance" back in performance management. SHRM-SIOP Science of HR White Paper Series, 1-25. Retrieved from https://pdri.com/wpcontent/uploads/2018/11/Putting-Performance-back-intoPerformanceManagement.pdf

O'Reilly, C. A., \& Anderson, J. C. (1980). Trust and the communication of performance appraisal information: The effect of feedback on performance and job satisfaction. Human Communication Research, 6, 290-298. doi: 10.1111/j.1468-2958.1980.tb00150.x

Organ, D. W. (1988). Organizational citizenship behavior: The good soldier syndrome. Lexington MA: Lexington Books.

Podsakoff, P. M., \& Farh, J. (1989). Effects on feedback sign and credibility on goal setting and task performance. Organizational Behavior and Human Decision Processes, 44, 45-67. doi: 10.1016/0749-5978(89)90034-4

Ryu, G., \& Hong, S.-W. (2019). The mediating effect of trust in supervisors in the relationship between constructive performance feedback and perceived fairness of performance appraisal. Public Performance E Management Review, 39, 1-18. doi: 10.1080/15309576.2019.1676274

Spector, P. E., Fox, S., Penney, L. M., Bruursema, K., Goh, A., \& Kessler, S. (2006). The dimensionality of counterproductivity: Are all counterproductive behaviors created equal? Journal of Vocational Behavior, 68, 446-460. doi: 10.1016/j.jvb.2005.10.005

Steelman, L. A., \& Rutkowski, K. A. (2004). Moderators of employee reactions to negative feedback. Journal of Managerial Psychology, 19(1), 6-18.

Tabachnick, B. G., \& Fidell, L. S. (2013). Using multivariate statistics (6th ed.). Boston, MA: Pearson.

Venables, W. N., \& Ripley, B. D. (2002). Modern applied statistics with $S$ (4th ed.) New York, NY: Springer.

Warnes, G. R., Bolker, B., Lumley, T., \& Johnson, R. C. (2018). gmodels: Various $\mathrm{R}$ programming tools for model fitting. $\mathrm{R}$ package version 2.18.1. Retrieved from https://CRAN.R-project.org/package=gmodels 